\title{
Guided and focused slow atomic beam from a 2 dimensional magneto optical trap
}

\author{
Yoann Bruneau ${ }^{1}$, Guyve Khalili ${ }^{1}$, Pierre Pillet ${ }^{1}$, and Daniel Comparat ${ }^{1}$ \\ Laboratoire Aimé Cotton, CNRS, Université Paris-Sud, ENS Cachan, Bât. 505, 91405 Orsay, France
}

Received: / Revised version: March 6, 2014

\begin{abstract}
We report experimental results which demonstrate how a single laser can be used to both accelerate and guide caesium atoms initially trapped in a two dimensional magneto-optical trap (2DMOT). The atomic beam size and velocity can be modified using both the laser power and detuning as parameters. In particular, using a $750 \mathrm{~mW}$ laser detuned $10 \mathrm{GHz}$ from resonance, we demonstrate that it is possible to focus an atomic beam to a size of $0.4 \mathrm{~mm}$ as far as $60 \mathrm{~cm}$ from the output of the 2D-MOT with a flux on the order of $3 \times 10^{9}$ atoms $/ \mathrm{s}$.
\end{abstract}

PACS. 67.85.-d ultracold gases - 07.77.Gx source of atomic beams

\section{Introduction}

Atomic beams have emerged as the preferred method of transport thanks to their high flux and stability. The main difficulty faced with these sources is the control over beam parameters, such as the divergence, longitudinal velocity and flux, which often having no or only minimal tuning.

The manipulation of atomic beams started with the pioneering studies made by A. Ashkin et al. [1], who manipulated the divergence of a sodium atomic beam produced from an oven using dipole trapping. The dipole force can alter the beam divergence and ultimately can be used to focus the atomic beam. However the longitudinal and transverse velocities of the atoms are fixed by the temperature of the oven with no possibility of control. For the case of caesium, we mention Ref. [2], where the authors demonstrated that it is possible to create a bright beam by compressing an effusive Cs beam. However, the experimental apparatus affects only the slowest atoms without controlling their axial velocities. Moreover, the atomic beam still diverges in free space. It is possible to control the longitudinal velocity by using a Zeeman deccelerator $[3,4]$, however this produces a beam with a non-negligible divergence, and only a small percentage of the atoms are slow down.

In order to have better control of the beam, cold atoms can be used as the source and thus the atomic beam obtained in this case has a narrow velocity spread. Atomic beams from cold atoms can be obtained by the pyramidal MOT [5,6], the conical mirror funnel [7] and the low velocity intense atomic source (LVIS) [8-10]. Using the now standard two-dimensional MOT [11-26], high flux, up to $10^{11}$ atoms/s can be obtained. However, the cold atomic beam expands radially once the atoms have left the two-dimensional cooling region, since there is no more transversal cooling. This kind of system is of major interest for atom interferometry, atom optics and precision atomic spectroscopy or even quantum optics applications, such as EIT, entangled photon pair generation, optical quantum memory, and quantum information processing [27]. One typical application is the loading of a 3D magnetooptical trap (MOT) in a ultra-high vacuum environment which allows for the reduction of interactions and collisions with the environment and thus increases the lifetime of the trap. In our group we are interested by another application: the transport of a collimated beam of ceasium atoms far from the MOT magnetic fields in order to be ionized to create a beam of unprecedented brightness and mono-energetic ion or electron beam $[28,11]$.

As demonstrated here, a 2D-MOT with the addition of dipole focusing facilitates atomic transport whilst reducing the beam divergence and providing a control over the beam size. This work is the continuation of the work performed in Ref. [29], where atoms were pushed and guided from a 3D-MOT to load another 3D-MOT. In the present case, by using a similar focusing and guiding technique, but now with a 2D-MOT, we reach a higher flux. The technique allows to transport atoms over large distances while maintaining the high density of the beam. We demonstrate that the atomic beam follows the laser beam shape along its propagation, and especially that focusing of the beam is possible.

We will first describe our experimental setup. Then, we will describe the experiment performed with the dipole pushing laser to guide the atoms. 


\section{Experimental results}

The 2D-MOT apparatus is available from SYRTE (Observatoire de Paris) and a detailed description can be found in Ref. [30].

Fig. 1 shows a schematic of the experiment. A cooling laser red-detuned $10 \mathrm{MHz}(\simeq-2 \Gamma)$ from the $6 S_{1 / 2}(F=$ $4) \rightarrow 6 P_{3 / 2}\left(F^{\prime}=5\right)$ resonance is separated into 3 beams which are retro-reflected through a glass cell, creating 3 regions of cooling aligned along the atomic beam propagation axes. Each region measures $2 \mathrm{~cm}$ along the atomic beam axis and $1 \mathrm{~cm}$ in the other dimensions, and the regions are separated by $1.25 \mathrm{~cm}$. A repumping laser, locked on the $6 S_{1 / 2}(F=3) \rightarrow 6 P_{3 / 2}\left(F^{\prime}=4\right)$ transition is sent along one dimension. The cell is surrounded by four magnetic coils which create a quadrupole field gradient of 0.2 $\mathrm{T} / \mathrm{m}$. A differential pumping section separates the $2 \mathrm{D}$ MOT chamber from the detection chamber with a $1.5 \mathrm{~mm}$ diameter aperture. A Ti:Sa pushing laser is applied along the beam axis with a maximum power of $750 \mathrm{~mW}$ at 852 $\mathrm{nm}$. The specifications, such as power, detuning and waist of this laser influence the atomic beam in terms of flux and divergence, and are the subject of this paper.

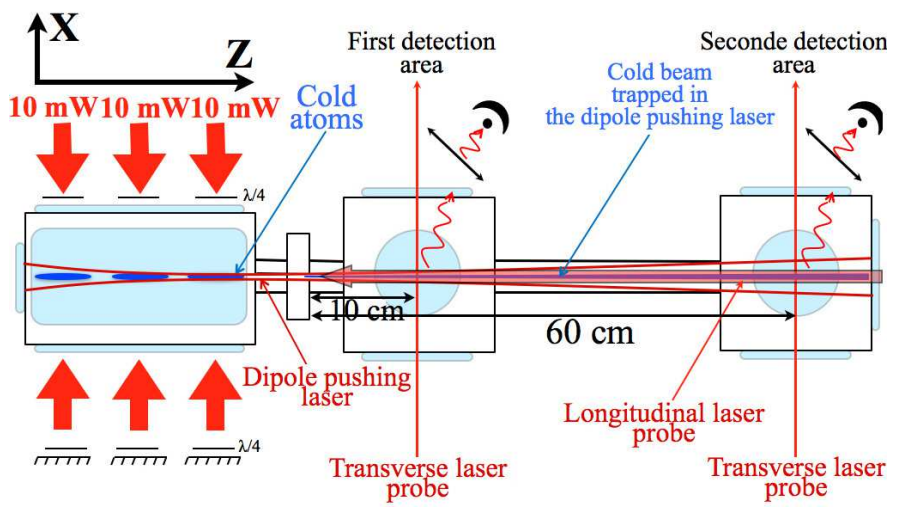

Fig. 1. Schematic of the experiment; on the left, the 2D-MOT with the 3 clouds of cold atoms; on the right, the 2 area of detection; the atomic beam properties are extracted from fluorescence measurements, that are performed $10 \mathrm{~cm}$ and $60 \mathrm{~cm}$ far from the 2D-MOT by the use of two transverse laser beam probes and a longitudinal probe.

Detection and characterization of the atomic beam are achieved via fluorescence measurements in two areas, respectively $10 \mathrm{~cm}$ and $60 \mathrm{~cm}$ far from the output of the 2D-MOT, where a probe laser beam is send orthogonally to the atomic beam. This configuration avoids shifts due to Doppler effect. Once the atoms have left the cooling region, there is no repumping laser and the atoms decay to the $\mathrm{F}=3$ hyperfine state and the effect of the pushing laser is negligible. However this implies that we need to add some repumping beam in probe beams in order to improve detection efficiency by having atoms cycling on the $6 S_{1 / 2}(F=4) \rightarrow 6 P_{3 / 2}\left(F^{\prime}=5\right)$ transition.

By scanning spatially the transverse probe with power below the saturation intensity, and collecting the fluorescence light by lenses and a calibrated photodiode, we can measure the shape and the density of the atomic beam. Indeed, by taking into account the waist of the transverse probe (of $1.3 \mathrm{~mm}$ in the first area and $0.75 \mathrm{~mm}$ in the second one) it is straightforward to extract the size of the atomic beam, which is found to have a nearly Gaussian spatial distribution. To extract the atomic beam size, we remove the waist size of the probe laser from the raw signal which is a convolution between the Gaussian probe laser and the atomic beam. In this article we always define the "size" of the atomic beam as the $(4 \sigma) 1 / e^{2} \mathrm{di}$ ameter of the beam. Except for very large laser beam size (more than few $\mathrm{mm}$ ) where the depth of field of our optical system reduces the amount of collected light, the atomic beam size is given typically within $15 \%$ accuracy. Obtaining quantitative results for the atomic beam density with fluorescence technique can be challenging due to absorption, diffusion or optical pumping effects. Consequently the absolute values for the peak atomic density $n_{0}$ and the atomic flux $F$ cannot be considered to be given accurately (the values are probably correct only within 50\%). Nevertheless all values are affected by the same systematic factors, such as collection light efficiency, thus relatives values for the integrated fluxes can be considered to be accuratly given within $15 \%$. The flux $F$ is calculated from $F=2 \pi \sigma^{2} n_{0} v$, where $v$ is the longitudinal mean speed of the atoms. It is extracted from the velocity distribution of the beam obtained using the Doppler effect by scanning the frequency of a longitudinal probe, and collecting the fluorescence as performed previously when a transverse probe was used. For accurate measurement we found it was important to use a weak longitudinal probe in order to not scatter too may photons which can lead to a modification of the velocity of the atoms during their travel.

Initially, we wanted to verify the specifications for Cs atoms comparing to those providing by SYRTE for Rb. With a power of $3 \mathrm{~mW}$ for the standard pushing laser collimated with a waist of $1.5 \mathrm{~mm}$, we measured a flux of $1.5 \times 10^{10}$ atoms/s along with a longitudinal velocity of $25 \mathrm{~m} / \mathrm{s}$. The atomic size is $7.6 \mathrm{~mm}$, in the second area (60 $\mathrm{cm}$ from 2D-MOT). We deduce a beam divergence of $2 \theta=11.2 \mathrm{mrad}$ which is in reasonable agreement with a transverse velocity given by the Doppler limit of $0.1 \mathrm{~m} / \mathrm{s}$ for $T=125 \mu \mathrm{K}$. These measurements agree with the specifications provided by the manufacturer, but now for Cs atoms. Further study of the atomic beam velocity versus the power, detuning or even waist of the pushing beam can be performed [17-21,23-25], but, one conclusion of these studies is that it does not seem possible to concentrate the flux of an atomic beam with a simple pushing laser due to the large beam divergence.

In an attempt to ameliorate the beam divergence produced with a simple pushing laser, we used a high-power off-resonant laser to both push and trap the beam as demonstrated in Ref. [29] in the case of a 3D-MOT.

In a first experiment, we choose the dipole pushing laser with a waist of $1.2 \mathrm{~mm}$ and a Rayleigh length of 5 $\mathrm{m}$, implying that the laser is collimated.

In addition to the pushing effect due to the radiation pressure the laser produced a dipole trap allows guid- 


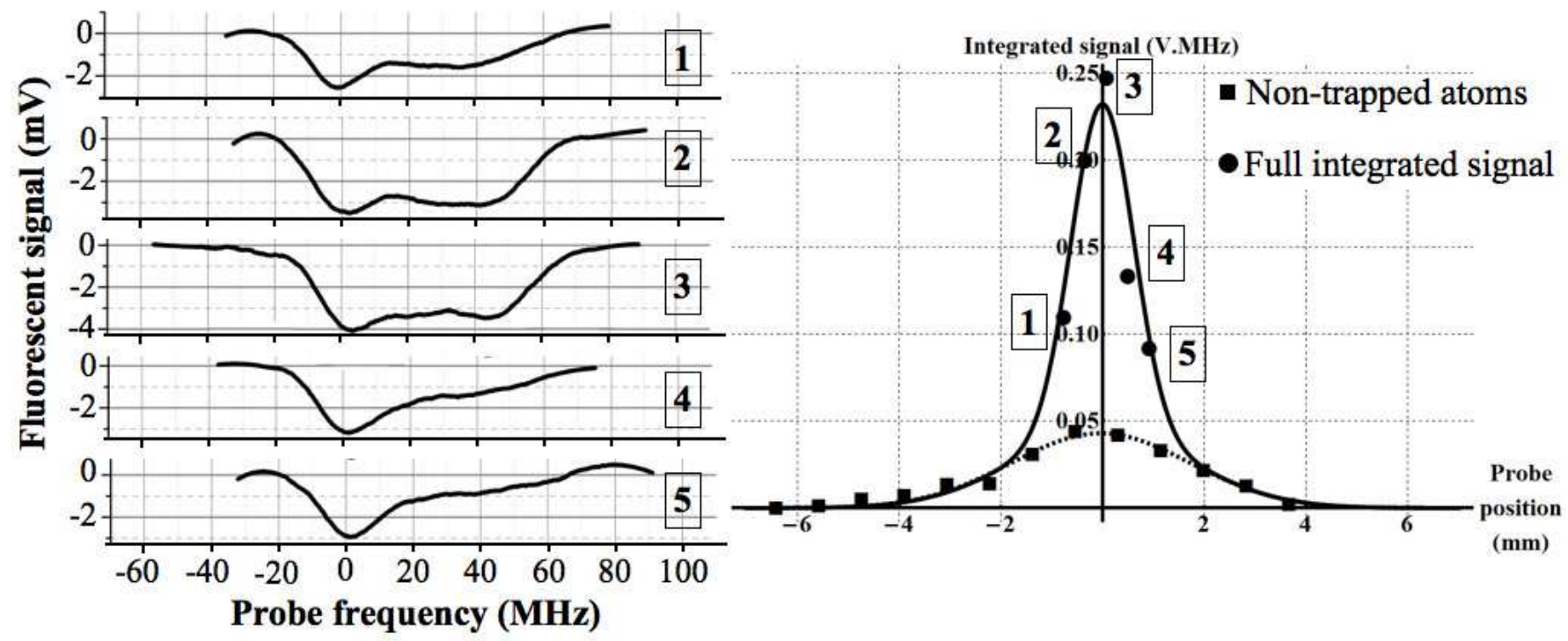

Fig. 2. Spatial cuts of the atomic beam for different positions of the transverse probe obtained $10 \mathrm{~cm}$ from the output of the MOT. A power of $750 \mathrm{~mW}$ and a detuning of $-10 \mathrm{GHz}$ was set for the dipole pushing laser. Left, raw signals obtained by scanning the detuning of the probe ; there are 2 peaks for each signals, the resonant signal on the left part and the blue shifted signal on the right part. Right, experimental points obtained by integrated the fluorescent signal for different positions of the probe ; square points present the integration of the resonant signal of the raw signal and stand for the non-trapped atoms (the dashed curve is a fit of these points) ; round points present the full integrated raw signals and stand for the entire beam of trapped and non-trapped atoms (the plain curve is a gaussian fit of the points).

ing of the atoms, provided that the trap depth is large enough. However, this makes measurement of the atomic beam parameters more involved because atoms inside the dipole trap are shifted out of resonance by the AC stark shift. The shift depends on the depth of the dipolar potential, which is dependent on the power and detuning of the dipole laser and on the distribution of the atoms in the trap [32]. On the contrary non-trapped atoms are obviously not shifted and, for a transverse probe, have all the resonance frequency of the transition $F=4 \rightarrow F^{\prime}=5$. Consequently, in order to separate the trapped atoms, from non trapped atoms we need to scan the probe detuning for each position of the probe. Fig. 2 (left panel) shows such raw signals obtained for 5 different vertical positions of the probe. They are taken in the first area of detection for a Ti:Sa power of $750 \mathrm{~mW}$ and a detuning of $-10 \mathrm{GHz}$. We clearly see the 2 peaks: one representing the atoms which are not trapped and not sensitive to the dipole pushing laser, and the second peak being blueshifted from the first signal due to the AC Stark shift. This interpretation is confirmed by the fact that for smaller detuning, the intensity of the second peak decreases but its width increases, which is expected because the dipole trap is deeper and closer to resonance the atoms are more heated and so expand in the trap.

In order to have a more quantitative measurement, for each signal, that is, for each position of the probe, we extract the trapped and non trapped part. First by fitting a Lorentzian with fixed linewidth (natural linewidth plus the laser linewidth) to the non-trapped atoms peak, we can estimate the proportion of non-trapped atoms (Fig. 2 , square points). Second, we integrate the entire signal (both the trapped and non-trapped atoms) which now is proportional to the total number of atoms present in the area. Fig. 2 (right panel) shows both results, the trapped part and the fully integrated part as a function of probe position. The figure clearly indicates that a $8 \mathrm{~mm}$ large and dilute cloud of non trapped atoms surround the trapped atomic beam. A great part of the atoms are trapped and the trapped atomic beam is concentrated in a small area in the center of the pushing laser.

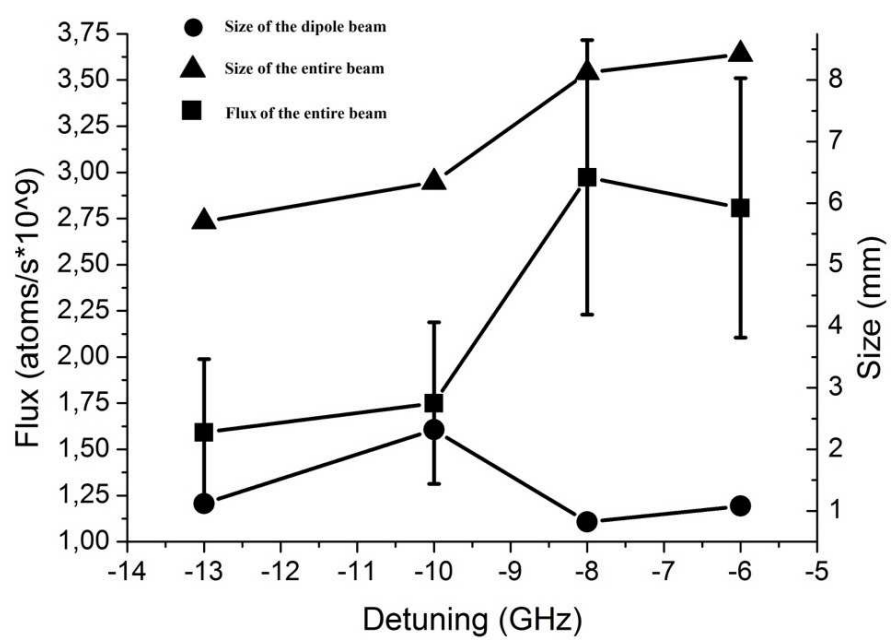

Fig. 3. Evolution of the flux and the size (the accuracy is 0.1 $\mathrm{mm}$ ) for the entire atomic beam and for the dipole trap for different detuning of the pushing laser at a constant power of $750 \mathrm{~mW}$; results obtained $10 \mathrm{~cm}$ far from the output of the 2D-MOT. 
Using these data, the atomic beam flux and size, still measured in the first area, are shown in Fig. 3. We see that it is possible to push nearly $3 \times 10^{9}$ atoms $/ \mathrm{s}$. With the dipole pushing laser, the flux is conserved from the first area to the second one, but is slightly lower than the flux obtained when using the standard pushing laser. This can be explained by the fact that the dipole pushing laser modifies the cooling process in the MOT due to the $\mathrm{AC}$ Stark shift.

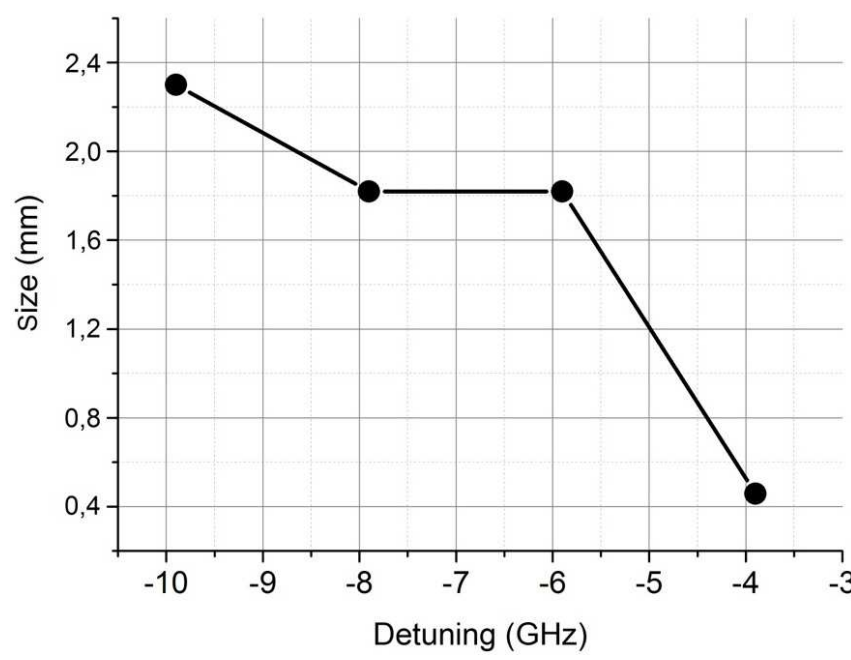

Fig. 4. Evolution of the size of the atomic beam, measured 60 $\mathrm{cm}$ far from the 2D-MOT, for different detuning of the pushing laser at a constant power of $750 \mathrm{~mW}$.

We now switch to studies performed using the second area of detection, $60 \mathrm{~cm}$ far from the 2D-MOT. This allows us to study in more detail the atomic beam divergence. The raw signals are simpler because of the presence of a single peak. Indeed, the beam is not "polluted" by non trapped atoms since only trapped atoms reach this second zone. We measured the size of the atomic beam in the second area as a function of the laser detuning and the results are shown in Fig. 4. As one would expect, the size of the atomic beam is lower than the previous results obtained with the standard pushing laser. It does not exceed $2.4 \mathrm{~mm}$ and is contained in the area of the pushing laser. As expected, the size decreases with the detuning when the depth of the trap increases. Under the same conditions, we measure with the longitudinal probe the velocity of the atoms. Fig. 5 shows the mean velocity obtained as a function of different detuning values. We see that the velocity increases when the detuning is closer to the resonance, which is consistent with our expectations, as atoms absorb more photons and are pushed more efficiently when the frequency is closer to the resonance.

Inside the 2D-MOT, the atoms can be heated as they are accelerated by the pushing laser. However, the trapping lasers temper this effect, allowing atoms to become trapped inside the pushing laser. Outside of the MOT, there is no repumping laser and the atoms are rapidly pumped towards the fundamental $F=3$ state. Thus the

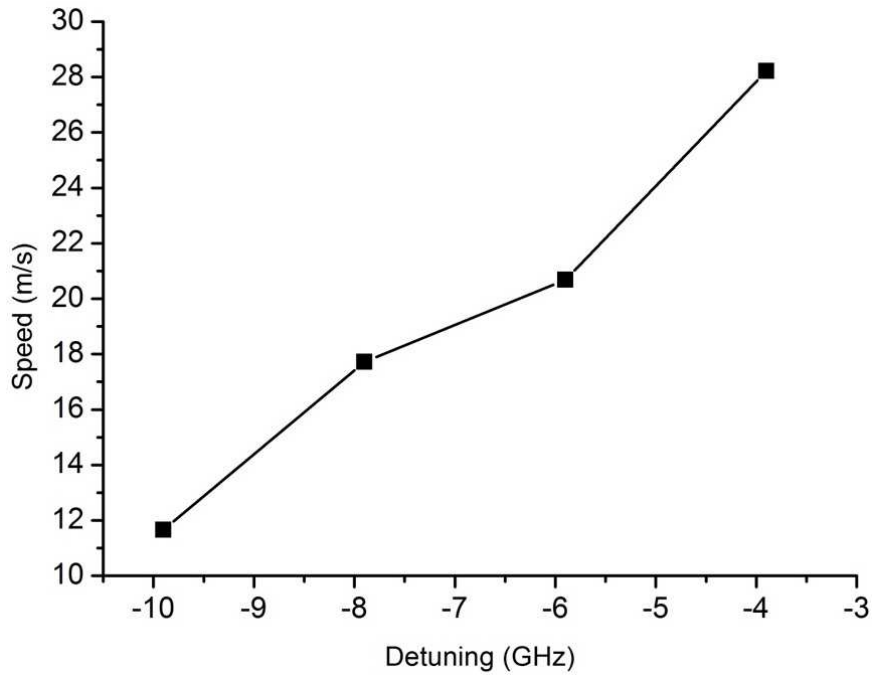

Fig. 5. Evolution of the speed of the atoms $60 \mathrm{~cm}$ from the output of the 2D-MOT versus the laser detuning for a power of $750 \mathrm{~mW}$.

detuning is decreased by $9.2 \mathrm{GHz}$ compared to the detuning in the 2D-MOT area which considerably reduces the effect of the pushing force and in turn the heating, whereas the dipole force is less affected. This allows atoms to be guided far from the output of the 2D-MOT without to much heating. Indeed, for an atom in the $F=3$ state, the scattering rate is 6400 photons.s ${ }^{-1}$ at a detuning of $-10 \mathrm{GHz}$. This implies that the temperature of the beam increases by $230 \mu \mathrm{K}$ during the $60 \mathrm{~cm}$ of travel. However, for large detunings, the depth of the trap is small $(175 \mu \mathrm{K}$ at a detuning of $-10 \mathrm{GHz}$ for $750 \mathrm{~mW}$ ) and gravity acts to decrease the trap depth. As shown in Fig. 4, the atomic beam size is equal to the size (that is twice the waist) of the pushing laser, implying that the atoms are at the edge of the trap, leading to a loss of atoms from the trap. In Fig. 6 , we see this effect, as the shape of the signal is elongated in the direction of gravity. To improve this, one requires a deeper trap, for which there are 2 solutions in this configuration of the pushing laser: increasing the trapping laser power or reducing the detuning. But both solutions would lead to heating. Another solution is to focus the pushing laser after the MOT, which has the additional benefit of beam focusing as well as beam guiding.

To investigate the effect of dipole focusing, we set the focal point of the dipole pushing laser after the 2D-MOT between the 2 detection areas. The position of the waist is now $30 \mathrm{~cm}$ before the second detection area. At this position, the waist of the pushing laser is $240 \mu \mathrm{m}$ and the effect due to the gravity is now negligible. Along the propagation, the waist $w(z)$ of the pushing laser changes and the condition to keep the atoms in the dipole potential is the adiabatic condition $\left(v(z) / \omega^{2}\right)(d \omega / d z) \ll 1$ where $\omega$ is the angular frequency of the oscillations of the atoms in the dipole trap. In the harmonic approximation, $\omega=\sqrt{2 k_{B} T /\left(w(z)^{2} m\right)}$ where $m$ is the mass of the Cs atoms, $k_{B}$ the Boltzmann constant. The condition becomes: $-v(z)(d w(z) / d z) \sqrt{m /\left(2 k_{B} T\right)} \ll 1$. With a de- 


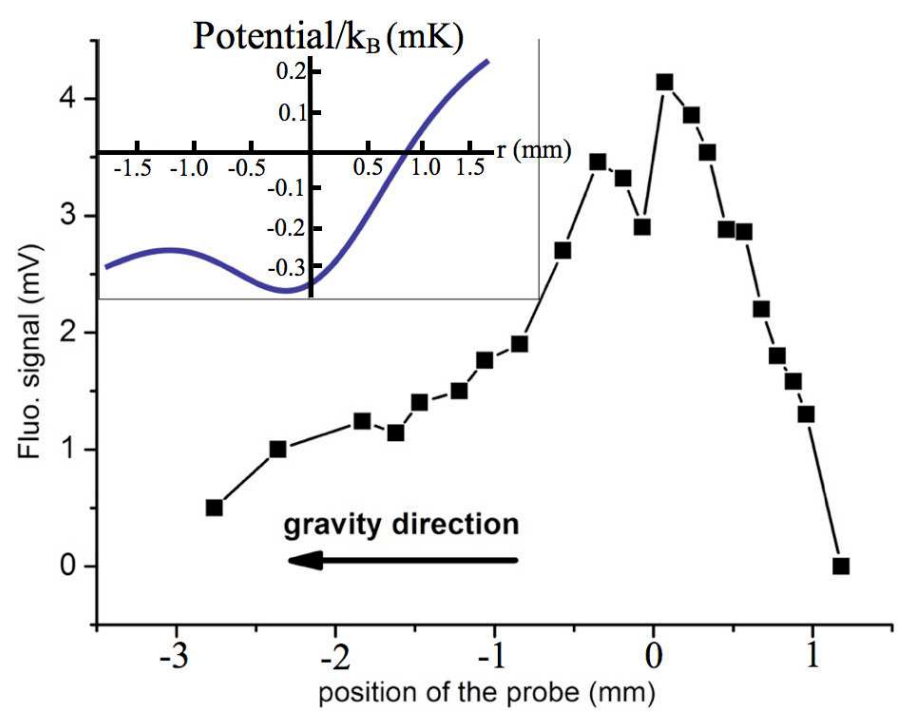

Fig. 6. Shape of the atomic beam, $60 \mathrm{~cm}$ from the output of the $2 \mathrm{D}-\mathrm{MOT}$, for $-10 \mathrm{GHz}$ and $750 \mathrm{~mW}$; inset : potential depth as function of $r$ (vertical direction).

tuning of $-10 \mathrm{GHz}$ and a power of $750 \mathrm{~mW}$, the mean velocity of the atoms is approximately $12 \mathrm{~m} / \mathrm{s}$ (from Fig. 5) allowing a maximum modification of the waist size $d w(z) / d z$ of $0.11 \mathrm{~mm} / \mathrm{cm}$ at $\mathrm{T}=125 \mu \mathrm{K}$. Our optical setup results in a waist modification of $0.0057 \mathrm{~mm} / \mathrm{cm}$, meaning the adiabatic condition is respected. Indeed, we found that the atomic flux of trapped atoms is conserved from the first to the second zone of detection. Fig. 7 shows the experimental points obtained in the second area of detection by scanning the beam with the transverse probe. The flux of $10^{9}$ is confirmed by integrating the signal of Fig. 7. We found a size of the atomic beam of $0.4 \mathrm{~mm}$, which corresponds to the waist of the pushing laser. These result proves that not only have we managed to guide the atomic beam, but in addition focus the beam.

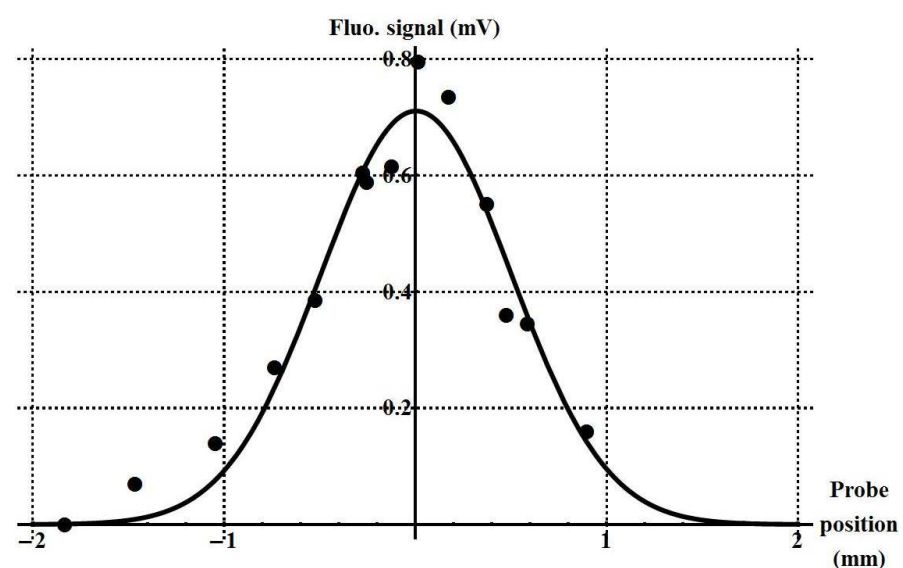

Fig. 7. Size of the atomic beam in the second area of detection in the second experiment ; points are experimental datas and the curve is a fit of experimental points ; the signal is the convolution of the waist probe 1.3 mmand the atomic beam shape ; thus, the true atomic beam size is $0.4 \mathrm{~mm}$.

\section{Conclusion}

This article shows a simple and robust experiment which is able to modify the divergence of an atomic beam created by a 2D-MOT by changing the detuning, the power and the size of the pushing laser. We note that Ref. [33] presents very similar results, but using a blue detuned Laguerre-Gaussian "doughnut" pushing laser beam. They also achieve a guiding of $100 \%$, but this experiment with $\mathrm{Rb}$ atoms hasn't yet demonstrated beam focusing.

In our case, with a high power and far red detuned pushing gaussian laser beam, we can push, trap and even focus the atoms in a small area. This allows the transport atoms to a precise position. We were able to transport more than $10^{9}$ atoms/s over $60 \mathrm{~cm}$ with a controllable velocity. This flux was ultimately limited by the available laser power for cooling. With increased power, a higher flux should be achieved. Furthermore, to improve the cooling effect, we can probably adjust the frequency of the cooling lasers to compensate for the AC stark shift [23]. Another solution could be to use 2 cooling lasers with 2 different frequencies : one frequency locked on the transition of the atoms when they are outside the dipole pushing laser and the other frequency locked on the transition shifted by the Stark effect.

Acknowledgements : The research leading to these results has received funding from the European Research Council under the grant agreement n. 277762 COLDNANO. We thank Andrew McCulloch for the proofreading of the article.

\section{References}

1. J. E. Bjorkholm, R. H. Freeman, A. Ashkin, and D. B. Pearson, Phys. Rev. Lett. 41, 1978, 1361.

2. J. Yu, J. Djemaa, P. Nosbaum, and P. Pillet, Opt. Commun. 112, 1994, 136.

3. W. Phillips and H. Metcalf, Phys. Rev. Lett. 48, 1982, 596.

4. H.J. Metcalf and P. van der Straten, Laser Cooling and Trapping, Springer, 1999.

5. J.J. Arlt, O. Maragò, S. Webster, S. Hopkins, C.J. Foot. Opt. Comm., 157, 1998, 303.

6. J. M. Kohel, J. Ramirez-Serrano, R. J. Thompson, L. Maleki, J. L. Bliss, K. G. Libbrecht, J. of Am. Soc. B, 20, 2003, 1161.

7. K. H. Kim, K. I. Lee, H. R. Noh, and W. Jhe, N. Kwon, and M. Ohtsu. Phys. Rev. A, 64, 2001, 3402.

8. Z. T. Lu, K. L. Corwin, M. J. Renn, M. H. Anderson, E. A. Cornell, and C. E. Wieman, Phys. Rev. Lett., 77, 1996, 3331.

9. P. Cren, C. F. Roos, A. Aclan, J. Dalibard, and D. GuéryOdelin. Eur. Phys. J. D, 20, 2002, 107.

10. B. K. Teo, T. Cubel, G. Raithel, Opt. Comm. 212, 2002, 307.

11. B. Knuffman, A. V. Steele, and J. J. McClelland. J. of Appl. Phys., 114, 2013, 044303.

12. Z. T. Lu, K. L. Corwin, M. J. Renn, M. H. Anderson, E. A. Cornell, and C. E. Wieman, Phys. Rev. Lett. 77, 1996, 3331. 
13. K. Dieckmann, R. J. C. Spreeuw, M. Weidemuller, and J. T. M. Walraven, Phys. Rev. A, 58, 1998, 3891.

14. J. Schoser, A. Batär, R. Löw, V. Schweikhard, A. Grabowski, Y. B. Ovchinnikov, and T. Pfau, Phys. Rev. A 66, 2002, 023410.

15. R.S. Conroy, Y. Xiao, M. Vengalattore, W. Rooijakkers, M. Prentiss, Opt. Comm., 226, 2003, 259.

16. T. Lahaye, J. M. Vogels, K. J. Gnter, Z. Wang, J. Dalibard, and D. Gury-Odelin, Phys. Rev. Lett., 93, 2004, 093003.

17. J. Ramirez-Serrano, N. Yu, J. M. Kohel, J. R. Kellogg, and L. Maleki. Opt. Lett., 31, 2006, 682.

18. J. Catani, P. Maioli, L. De Sarlo, F. Minardi, and M. Inguscio, Phys. Rev. A 73, 2006, 033415.

19. S. Götz, B. Höltkemeier, C. S. Hofmann, D. Litsch, B. D. DePaola, and M. Weidemüller, Rev. Sci. Instr. 83, 2012, 073112.

20. N. Castagna, J. Guéna, M. D. Plimmer and P. Thomann, Eur. Phys. J. Appl. Phys. 34, 2006, 21.

21. T. G. Tiecke, S. D. Gensemer, A. Ludewig, and J. T. M. Walraven. Phys. Rev. A, 80, 2009, 013409.

22. X.-L. Wang, B. Cheng, B. Wu, Z.-Y. Wang, and Q. Lin. Chin. Phys. Lett., 28, 2011, 053701.

23. S. J. Park, J. Noh, J. Munn, Opt. Comm., 285, 2012, 3950.

24. J. R. Kellogg, D. Schlippert, J. M. Kohel, R. J. Thompson, D. C. Aveline, and N. Yu, Appl. Phys. B, 109, 2012, 61.

25. S. P. Ram, S. K. Tiwari, S. R. Mishra, and H. S. Rawat. Rev. of Sci. Instr., 84, 2013, 073102.

26. S. Dorscher, A. Thobe, B. Hundt, A. Kochanke, R. Le Targat, P. Windpassinger, C. Becker, and K. Sengstock. Rev. of Sci. Instr., 84, 2013, 043109.

27. S. Zhang, J. F. Chen, C. Liu, S. Zhou, M. M. T. Loy, G. K. L. Wong, and S. Du. A dark-line two-dimensional magnetooptical trap of $85 \mathrm{Rb}$ atoms with high optical depth. Rev. of Sci. Instr., 83, 2012, 073102.

28. L. Kime, A. Fioretti, Y. Bruneau, N. Porfido, F. Fuso, M. Viteau, G. Khalili, N. Šantić, A. Gloter, B. Rasser, P. Sudraud, P. Pillet, D. Comparat, Phys. Rev. A 11, 2013, 11.

29. E. Dimova, O. Morizot, G. Stern, C.L. Garrido Alzar, A. Fioretti, V. Lorent, D. Comparat, H. Perrin, and P. Pillet, Eur. Phys J. D, 42, 2007, 299.

30. Patrick Cheinet, Conception et Réalisation d'un Gravimètre à Atomes Froids, PhD thesis, 2006.

31. K. Jooya, N. Musterer, K. W. Madison, and J. L. Booth, Phys. Rev. A, 88, 2013, 063401.

32. R. Grimm, M. Weidemüller, and YB Ovchinnikov, Adv. At. Mol. Opt. Phys. 42, 2000, 95.

33. V. Carrat, C. Cabrera-Guitierez, M. Jacquey, J. W. Tabosa, B. Viaris de Lesegno, L. Pruvost, arXiv:1304.7243v1, 2013 (accepted in Opt. Lett.). 